
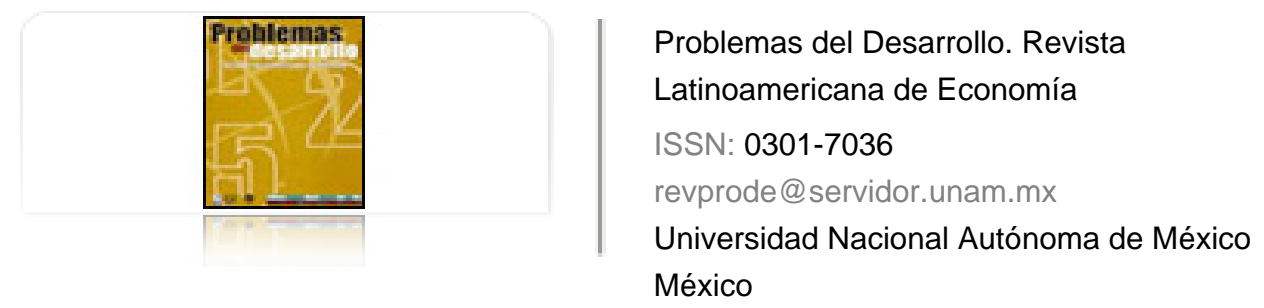

Nemiña, Pablo

ALCANCES DE LA REFORMA DE LA POLÍTICA DE FINANCIAMIENTO Y CONDICIONALIDAD DEL

FMI

Problemas del Desarrollo. Revista Latinoamericana de Economía, vol. 41, núm. 160, enero-marzo, 2010, pp. 39-66

Universidad Nacional Autónoma de México

Distrito Federal, México

Disponible en: http://www.redalyc.org/articulo.oa?id=11820132003

Cómo citar el artículo

- Número completo

- Más información del artículo

Página de la revista en redalyc.org

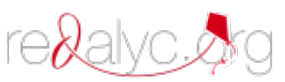

Sistema de Información Científica

Red de Revistas Científicas de América Latina, el Caribe, España y Portugal Proyecto académico sin fines de lucro, desarrollado bajo la iniciativa de acceso abierto 


\title{
ALCANCES DE LA REFORMA DE LA POLÍTICA DE FINANCIAMIENTO Y CONDICIONALIDAD DEL FMI
}

\author{
Pablo Nemiña*
}

Fecha de recepción: 14 de octubre de 2009. Fecha de aceptación: 6 de enero de 2010.

\section{Resumen}

En marzo de 2009, el FMI reformó su política de financiamiento y de condicionalidad, buscando alentar políticas contracíclicas y reducir la interferencia sobre la política local de los países prestatarios. Este trabajo procura analizar en qué medida la reforma estableció cambios concretos en la práctica, en función del objetivo planteado. Se estudian los acuerdos aprobados en el año transcurrido desde el estallido de la crisis, y se consideran la magnitud y el cronograma de desembolsos, así como las condicionalidades por área, tipo y grado de exigencia. Luego, se comparan los resultados antes y después de la reforma. Se propone que aunque la reforma logró el objetivo de promover políticas contracíclicas en relación con la política financiera, el alcance de la política de condicionalidad fue más limitado. Esto se verifica en la mayor exigencia de condiciones orientadas a ajustar el gasto y la continuidad en la ausencia de limitaciones a los movimientos de capital.

Palabras clave: FMI, créditos, condicionalidades, reforma, acuerdos.

* Sociólogo (UBA). Estudiante del Doctorado en Ciencias Sociales de la UBA. Becario CONICET con sede en el Instituto de Altos Estudios Sociales de la Universidad Nacional de San Martín. Correo electrónico: pablonemina@yahoo.com.ar

Versiones preliminares del presente trabajo fueron presentadas en un seminario realizado en el marco del Programa Interdisciplinario para el Seguimiento del Desarrollo y los Efectos de la Crisis en el Orden Económico Mundial (Pisco), en el Instituto de Desarrollo Humano, Universidad Nacional de General Sarmiento, el 20 de agosto de 2009; así como en la conferencia Reforming the Bretton Woods Institutions, en el Danish Institute for International Studies y el Danish Foreign Ministry, Copenhague, el 16 y 17 de septiembre de 2009.

El autor agradece los valiosos comentarios de Ricardo Aronskind, Mariela Bembi, Ana Castellani, Martín Schorr, así como de los tres evaluadores anónimos. Naturalmente, se los exime de toda responsabilidad en cuanto a los errores u omisiones existentes. 


\begin{abstract}
In March, 2009, the IMF reformed its financing and conditionality policy, seeking to encourage counter-cyclical policies and to reduce interference in local policy for borrowing countries. This study seeks to analyze to what extent the reform made concrete changes in practice, as a function of the established target. The agreements approved during the year are studied from the outbreak of the crisis, and the magnitude of the disbursements and a calendar for them, plus the conditionalities by area, type and grade of demand are considered. After that, the results before and after the reform are compared. It is proposed that although the reform achieved the objective of promoting counter-cyclical policies related to financial policy, the scope of the conditionality policy was more limited. This is verified by the greater demand for conditions oriented to the adjustment of spending and a continued lack of limitations on capital movements.

Key words: IMF, credits, conditionalities, reform, agreements.
\end{abstract}

\title{
Résumé
}

En mars 2009, le FMI a réformé sa politique de financement et de conditions de prêt, dans le but de fomenter des politiques anticycliques et réduire l'influence sur la politique locale des pays délivreurs de prêts. Ce travail essaye d'analyser dans quelle mesure la réforme a généré, dans la pratique, des changements concrets et conformes au but poursuivi. Ils sont étudiés les accords approuvés dans l'année écoulée depuis que la crise a éclaté, et considérés la magnitude et le chronogramme des déboursements ainsi que les conditions de prêt selon le secteur, le type d'exigence et son degré. Ensuite, il est fait la comparaison entre les résultats avant la réforme et après celle-ci. Il est postulé que, bien que la réforme ait atteint l'objectif de promouvoir des politiques anticycliques en rapport avec la politique financière, la portée de la politique de prêt a été plus limitée. Ceci est confirmé par l'imposition de conditions de prêt plus rigoureuses orientées à ajuster la dépense, et par le fait que les mouvements de capitaux ne soient toujours pas limités.

Mots clés: FMI, crédits, conditions de prêt, réforme, accords.

\section{Resumo}

Em março de 2009, o FMI reformulou sua política de financiamento e de condicionalidade, buscando alentar políticas contracíclicas e reduzir a interferência na política local dos países prestatários. Este trabalho procura analisar em que medida a reforma estabeleceu mudanças concretas na prática, em função do objetivo colocado. Estudam-se os acordos aprovados no ano transcorrido a partir do estouro da crise, e considera-se a magnitude e o cronograma de desembolsos, bem como as condicionalidades por área, tipo e grau de exigência. Em seguida comparam-se os resultados antes e depois da reforma. Propõe-se que apesar da reforma ter logrado o objetivo de promover políticas contracíclicas em relação à política financeira, o alcance da política de condicionalidade foi mais limitado. Isto se verifica na maior exigência de condições orientadas para ajustar o gasto e a continuidade na ausência de limitações aos movimentos de capital.

Palavras-chave: FMI, créditos, condicionalidades, reforma, acordos.

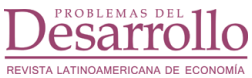

Vol. 41, núm. 160, enero-marzo / 2010 


\section{Introducción}

ace no más de un año, el Fondo Monetario Internacional (FMI) se encontra-
ba inmerso en una crisis comparable sólo a la que enfrentó durante la déca-
da de 1970, con la desaparición del patrón dólar-oro. Alentados por el ciclo de crecimiento económico del último lustro, los países en desarrollo -tradicionales prestatarios del organismo- habían acumulado reservas suficientes para evitar recurrir al Fondo ante una turbulencia externa. Las fallidas intervenciones del organismo en crisis anteriores (en especial en las del Sudeste asiático y Argentina), la baja magnitud de sus recursos y las intrusivas condicionalidades que acompañaban a sus créditos, desalentaban el interés por solicitar su financiamiento. Deslegitimado, y afectado por un creciente déficit operativo, se debatía entre reformarse o desaparecer.

Pero ante el estallido de la crisis financiera en septiembre de 2008, el FMI fue reposicionado como un importante actor en el escenario internacional. Además de resaltar la función de liderazgo que debía ocupar en la tarea de monitorear la estabilidad del sistema financiero internacional y la economía mundial para minimizar el riesgo de una crisis futura, el G20 anunció un aumento sustancial de sus recursos para hacer frente a las necesidades financieras de los países afectados por la crisis (G20, 2008a; 2008b).

El aumento de los recursos se inscribió en el marco del consenso político alcanzado entre las naciones del G20, respecto de alentar la aplicación de políticas económicas contracíclicas a escala global. Mientras los países desarrollados reducían la tasa de interés y elevaban el nivel de gasto, el financiamiento del FMI debía contribuir a mitigar el efecto recesivo del shock externo en los países en desarrollo, los cuales poseen una limitada capacidad para recurrir a políticas fiscales y/o monetarias expansivas.

Ante la desconfianza de los países en desarrollo hacia sus recetas y el interés de los países centrales por aumentar los créditos multilaterales, en marzo de 2009 el Fondo aprobó una reforma de su política de financiamiento y condicionalidad, mediante la cual aumentó la magnitud de sus desembolsos, y simplificó y flexibilizó las condicionalidades. Por medio de ella buscaba renovar el atractivo de sus líneas de crédito, adecuándose al renovado consenso político internacional.

En este sentido, el presente artículo procura analizar en qué medida la reforma logró transformar en la práctica la política crediticia y de condicionalidad del FMI, en función del objetivo de alentar políticas contracíclicas y reducir la interferencia sobre la política local de los países prestatarios.

Se propone que aunque la reforma logró el objetivo de posibilitar políticas contracíclicas en relación con la política financiera -ya que a partir de su aprobación se 
incrementaron los créditos-, el alcance ha sido mucho más limitado respecto de la política de condicionalidad, dado que se observan novedades junto a continuidades en aspectos prioritarios. Siguiendo este último punto, el Fondo avanzó en la concentración de las condiciones en sus áreas de responsabilidad principal y redujo de forma limitada la presión sobre los procesos políticos locales, mediante la eliminación de ciertas condicionalidades. No obstante, la continuidad en la orientación ortodoxa de sus condicionalidades en áreas centrales como la política cambiaria, de endeudamiento y de manejo del gasto público limita el espacio para la aplicación de políticas contracíclicas en los países que suscriben acuerdos. Esto se comprende toda vez que el FMI sigue acompañando sus desembolsos de condiciones que impiden restringir la salida de capitales, como la no aceptación de controles de capital o del aplazamiento en los pagos externos -aunque sea de forma temporal-, lo que profundiza el impacto del shock externo. De esta manera, aumenta la presión para que se lleven a cabo políticas fiscales y monetarias restrictivas -como recortes del gasto público o aumento de la tasa de interés- que aunque procuran evitar una caída brusca en el valor de la moneda nacional, agravan la contracción económica.

El desarrollo del artículo se organiza del siguiente modo: la segunda sección expone los aspectos principales de la reforma y el contexto económico en el que se enmarcó su aprobación. La tercera y cuarta secciones presentan la descripción y el análisis de la trayectoria seguida por las principales variables de las políticas crediticia y de condicionalidad, luego de la aprobación de la reforma. A fin de reflejar con mayor exactitud el alcance de las transformaciones en el FMI, los resultados se comparan con la trayectoria de las políticas mencionadas desde el estallido de la crisis económica hasta antes de la reforma, y en el caso de la política crediticia, con el comportamiento del Fondo durante la crisis del Sudeste asiático de 1997-1998. La quinta sección expone las consecuencias de la reforma en el FMI para los países de América Latina. Por último, se presentan las conclusiones.

\section{Historia y características de la reforma}

Una comprensión históricamente contextualizada de la aprobación de la reforma de la política de financiamiento y condicionalidad del FMI requiere considerar las características de la crisis financiera que empieza a manifestarse a comienzos de 2007 con la disminución de los precios de las viviendas en los EEUU y la consecuente caída de la cotización de los títulos respaldados por hipotecas subprime, y que estalla en septiembre de 2008 con la quiebra del banco de inversión Lehman Brothers (Arceo, Golonbek y Kupelian, 2009: 50).

\section{DeSRarrollo}


La actual crisis financiera se inició en el centro del capitalismo mundial y desde allí se expandió hacia el resto de las economías del globo (afectando en especial a Islandia y otros países de Europa del este). En este contexto, en la decisión de alentar mayores desembolsos por parte del FMI confluyeron: el interés de los EEUU por moderar la caída de la demanda global, el de las potencias europeas por evitar un estallido financiero en otros países de la Unión que afectara las inversiones de su sector privado ${ }^{1}$, también, el de los países en desarrollo por obtener financiamiento a bajas tasas de interés.

Para ello, se decidió triplicar los recursos del Fondo a US \$750 000 millones, mediante aportaciones directas de los países industrializados, la venta de una octava parte de sus reservas de oro (casi 13 millones de onzas troy) y la emisión de notas negociables en el sector financiero oficial. Las aportaciones directas se realizarán en el marco de acuerdos de préstamo ad hoc con cada país y posteriormente se incorporarán a los Nuevos Acuerdos para la Obtención de Préstamos (NAP) ${ }^{2}$, a fin de aumentar los recursos sin afrontar la discusión por la distribución de las cuotas. Esta última ha sido reclamada reiteradamente por los países en desarrollo, para que la estructura de poder del organismo refleje la nueva configuración de poder en la economía mundial (Torres, 2007: 447). Además, se aprobó una asignación general de Derechos Especiales de Giro (DEG) por US \$283000 millones, con el fin de ampliar la liquidez internacional.

Pero si la crisis incrementó la necesidad de financiamiento, para aumentar los créditos del organismo no sólo era necesario ampliar sus recursos, sino revertir la desconfianza de los países en desarrollo hacia sus recetas. Escepticismo sustentado en dos motivos: primero, el Fondo no estaba en condiciones de otorgar apoyo financiero significativo, de forma rápida y concentrada al inicio del acuerdo, que permitiera a las economías emergentes enfrentar con éxito la volatilidad de los capitales (Torres, 2008); segundo, a pesar de haber transcurrido cinco años desde la aprobación de los lineamientos de condicionalidad en 2002, no se veían mejoras en esta área. En espe-

1 En Islandia, por ejemplo, los créditos multilaterales buscaron, entre otros objetivos, mitigar el impacto de los problemas del sistema bancario sobre los depositantes, en gran parte ingleses y holandeses. En este sentido, funcionarios islandeses sospecharon que el FMI demoró la aprobación de la primera revisión del acuerdo, como resultado de la presión del Reino Unido y Holanda para que Islandia aceptara -como finalmente sucedió- otorgar compensaciones a los ahorristas afectados por las reestructuraciones bancarias (Ward y Parker, 31/7/09).

2 Los NAP son acuerdos de crédito entre el FMI y un grupo de países para suministrar recursos suplementarios por un monto de hasta US \$ 50000 millones, con el fin de evitar, o hacer frente, a una crisis que represente una amenaza para la estabilidad del sistema financiero internacional. 
cial, se señalaba que la orientación intelectual de las mismas seguía basándose en la ortodoxia liberal (Griesgraber, 2009: 182), el número de condicionalidades estructurales incluidas en los acuerdos con países de ingreso bajo -sus principales prestatarios durante ese periodo- no había disminuido, y una cuarta parte de las condiciones formaban parte de áreas ajenas a su expertise y políticamente sensibles como privatizaciones y liberalizaciones (Molina y Pereira, 2008: 4).

Cuadro 1

Aumento de recursos del FMI, al 27/9/09

(millones de dólares)

\begin{tabular}{|c|c|}
\hline Fuente de financiamiento & Monto \\
\hline Aportaciones directas de países & 421300 \\
\hline Unión Europea $^{1}$ & 178000 \\
\hline Japón & 100000 \\
\hline Canadá $^{\prime}$ & 10000 \\
\hline Noruega & 4500 \\
\hline EEUU & 100000 \\
\hline Suiza & 10000 \\
\hline Corea del Sur & 10000 \\
\hline Australia & 5700 \\
\hline Singapur & 1500 \\
\hline Chile & 1600 \\
\hline Suscripción de notas del FMI & 80000 \\
\hline China & 50000 \\
\hline Rusia & 10000 \\
\hline Brasil & 10000 \\
\hline India & 10000 \\
\hline Venta de oro ${ }^{2}$ & 14056 \\
\hline Total & 515356 \\
\hline
\end{tabular}

Se señalan en cursiva los países que aún no han hecho efectivo su compromiso.

${ }^{1}$ Hasta el momento efectivizó US $\$ 70000$ millones.

212966 millones de onzas troy a US \$1 084.1 por onza.

Fuente: Elaboración propia con base en IMF (2009b; 2009c) y bloomberg.com

Por ello, reposicionar al Fondo en su papel de prestamista en crisis financieras implicaba complementar el aumento de sus recursos con una revisión de sus políticas de crédito y condicionalidad (G20, 2009a).

Los antecedentes previos a la reforma permiten observar que si bien el aumento de los límites de financiamiento y la flexibilización de las condiciones eran temas que formaban parte de la agenda del organismo (en rigor, el primero con más claridad que el segundo), hasta el estallido de la crisis no se vislumbraban propuestas concretas de modificación. Por ejemplo, en marzo de 2008 el Directorio del Fondo

\section{Desarrollo}


llevó a cabo la revisión periódica de la adecuación de los límites de acceso al financiamiento y los dejó sin cambios (IMF, 2008a). Pero poco después del estallido de la crisis financiera, las propuestas de reforma comenzaron a cobrar vigor a un ritmo acelerado. Una semana después de la quiebra de Lehman Brothers, el Directorio discutió la función del Fondo como prestamista y planteó revisar -nuevamente- los límites de acceso, crear una nueva línea de crédito de rápido desembolso y avanzar en la reforma de la condicionalidad (IMF, 2008b). En enero de 2009 se acordó de forma preliminar aumentar los límites de acceso y, finalmente, el 24 de marzo se aprobó la reforma de las políticas de financiamiento y condicionalidad (IMF, 2009a).

La situación expuesta permite inferir que aunque el Fondo se mostraba un tanto dispuesto a introducir modificaciones, la confluencia de intereses entre los países centrales -resueltos a devolver al Fondo su función de prestamista en crisis financieras- y los países en desarrollo -conformes con la posibilidad de flexibilizar las exigencias para acceder al financiamiento del organismo-fue clave para conseguir la aprobación -en un plazo relativamente breve- de una reforma que incluyó modificaciones sustanciales. A continuación, se desarrollan las características más relevantes de la misma.

Respecto de la política de financiamiento pueden señalarse tres novedades. Primero, la renovación del menú de líneas de crédito, mediante la cual se eliminaron algunas de las menos usadas y se estableció la Línea de Crédito Flexible (LCF) ${ }^{3}$. Ésta ofrece financiamiento ilimitado (aunque se espera que no sea mayor al 1 000\% de la cuota de los países), con un largo plazo de repago (de tres años tres meses a cinco años), sin condicionalidades, y la posibilidad de ser usado tanto de forma precautoria como ante necesidades de balanza de pagos. Pero no todos los países pueden solicitarla, ya que está limitada a los que posean una base económica y un marco institucional estables, una muy sólida trayectoria de políticas y que, además, hayan sido evaluados positivamente en la revisión anual ${ }^{4}$. Segundo, se reforzó el

3 Fueron discontinuados el Servicio de Financiamiento Compensatorio (SFC), el Servicio de Complementación de Reservas (SCR), y el Servicio de Liquidez a Corto Plazo (SLC). El primero proveía financiamiento ante una caída brusca del precio de las exportaciones pero no había sido usado desde 1999, ya que en esos casos los países optaban por recurrir a los mercados de capital privado. El segundo ofrecía alto financiamiento, pero a muy corto plazo y con una elevada sobretasa que desalentaba su uso. El último, aprobado en octubre de 2008, fue reemplazado por la LCF. También se decidió desalentar el uso del Servicio Ampliado del FMI (SAF) para grandes desembolsos.

4 Los criterios relevantes a cumplir para la aprobación de la LCF incluyen: $a$ ) una posición externa sostenible, $b$ ) predominio de flujos privados en el financiamiento de la cuenta de capital, c) una trayectoria estable de acceso en términos favorables a los mercados de financiamiento 
Stand-By (SBA) -la línea de crédito tradicional- por medio de la duplicación de los límites de acceso normales ${ }^{5}$ (de 100\% a 200\% de la cuota por año y de 300\% a $600 \%$ de la cuota total). Tercero, y en relación con el punto anterior, se habilitó, en caso de que las condiciones económicas lo hicieran necesario o lo permitieran, la posibilidad de aumentar la concentración de los desembolsos al inicio del acuerdo y de reducir la frecuencia de las revisiones de trimestral a semestral.

En relación con la política de condicionalidad, se propuso una reforma orientada a adaptar las condiciones a los requerimientos de los países - de acuerdo con sus debilidades y fortalezas-, y a reducir el estigma asociado a su cumplimiento, basada en tres pilares: 1) reducir las condiciones tradicionales (ex post) en los acuerdos, mediante la mayor utilización de criterios de precalificación (condicionalidad ex ante); 2) eliminar los criterios estructurales de aplicación y monitorear el cumplimiento de las reformas estructurales en la instancia de revisión de los programas; 3) limitar la condicionalidad a sus áreas centrales de expertise, esto es, variables macroeconómicas financieras, monetarias, fiscales y cambiarias.

Para responder al interrogante acerca de en qué medida la reforma introdujo modificaciones sustanciales en las prácticas financieras y de condicionalidad del FMI, a continuación se describe y analiza la trayectoria seguida por las principales variables en las que la reforma introdujo cambios: magnitud y secuencia de los créditos comprometidos y desembolsados, distribución de las condicionalidades por área y tipo, y grado de exigencia de las mismas según área ${ }^{6}$. Como fue señalado, se analizan los resultados de la trayectoria de las variables mencionadas desde el estallido de la crisis económica en septiembre de 2008 hasta agosto de 2009 (fecha hasta la que existe disponibilidad de datos), distinguiendo entre el periodo anterior

internacional, $d$ ) una relativamente confortable posición de reserva cuando la LCF se solicita en base precautoria, $e$ ) sólidas finanzas públicas, incluyendo una posición de deuda pública sostenible, f) niveles de inflación bajos y estables, en el contexto de un marco de políticas monetaria y cambiaria sólidas, $g$ ) ausencia de problemas de solvencia en el sector bancario, $h$ ) supervisión efectiva del sector financiero e $i$ ) transparencia e integridad estadística.

5 El Fondo establece límites de acceso considerados normales para sus líneas de crédito, aunque puede autorizar desembolsos por encima de ellos, siempre que los países cumplan con los siguientes criterios: $a$ ) atravesar por una crisis de balanza de pagos, $b$ ) la deuda permanecerá en niveles sostenibles, $c$ ) existen expectativas de retornar prontamente a los mercados privados de capital y $d$ ) el programa posee altas oportunidades de éxito. De todas maneras, cuenta con la potestad de flexibilizar la aplicación de los criterios en el caso de que no se cumplan en su totalidad.

6 Debido al breve tiempo transcurrido desde la aprobación de la reforma, se dejó de lado la consideración del alcance de la simplificación de la estructura de cargos y costos, y la reducción de las frecuencias de las revisiones. 
y posterior a la reforma, con la finalidad de reflejar con la mayor exactitud los cambios y continuidades observados.

\section{Evidencia empírica acerca de la política financiera}

\subsection{Líneas de crédito y desembolsos}

Desde el estallido de la crisis, el Fondo aprobó 20 acuerdos, 17 SBA y tres en la nueva LCF. La primera conclusión es que el aumento de sus recursos se ha reflejado en un marcado crecimiento en la dimensión de los créditos comprometidos y, aunque en una magnitud menor, en los desembolsos.

\section{Cuadro 2}

FMI: Créditos aprobados, desembolsos y promedio por mes (sep. 2008 a ago. 2009) En millones de DE G. Acuerdos SBA y LCF

\begin{tabular}{|l|c|c|c|c|}
\hline & $\begin{array}{c}\text { Créditos } \\
\text { aprobados }\end{array}$ & Desembolsos & $\begin{array}{c}\text { Promedio créditos } \\
\text { aprobados por mes }\end{array}$ & $\begin{array}{c}\text { Promedio desembolsos } \\
\text { por mes }\end{array}$ \\
\hline Sep 08-ago 09 & 105697 & 26318 & 8808 & 2193 \\
\hline Prerreforma (sep 08- mar 09) & 38125 & 13459 & 5446 & 1923 \\
\hline Posrreforma (abr 09-ago 09) & 667572 & 12858 & 13514 & 2572 \\
\hline
\end{tabular}

Fuente: Elaboración propia con base en www.imf.org

Al comparar el promedio por mes antes y después de la reforma, se observa que el crédito comprometido se incrementó en casi una vez y media, y los desembolsos en casi un 35\%. Esta diferencia se entiende al discriminar entre créditos SBA y LCF. Los tres créditos LCF son de gran magnitud, pero hasta el momento no fueron utilizados por los países solicitantes (México, Colombia y Polonia).

A fin de poner en perspectiva el alcance de este cambio, se presenta una comparación de la magnitud de los créditos aprobados y los desembolsos durante el primer año a partir del estallido de la presente crisis y la del Sudeste asiático, el ejemplo más reciente de una crisis financiera comparable a la actual en términos de la intervención del FMI (véanse datos detallados en los cuadros A.1 y A.2 del anexo) 7 . Aunque podría argumentarse que algunas diferencias limitarían el alcance de la comparación (la crisis

7 Aunque el FMI participó activamente en la crisis 2001-2002 -que afectó especialmente a Turquía, Brasil y Argentina-, se decidió no incluirla en la comparación debido a que no puede identificarse un momento como punto de inicio de la misma. Aunque debatible, existe consenso en considerar la devaluación del bath tailandés en julio de 1997 y la quiebra de Lehman Brothers en septiembre de 2008 como el inicio de las crisis escogidas. 
actual es más profunda y se inició en el centro del capitalismo mundial), ambos casos comparten el hecho de ser claras referencias de crisis financieras en cuanto fenómeno económico ${ }^{8}$, y la existencia de una activa participación del FMI -mediante el otorgamiento de créditos y la exigencia de medidas de política económica- desde que la crisis se desencadenó.

Cuadro 3

FMI: Créditos aprobados y desembolsos; Relación con cuotas (jul. 97 - jun. 98 y sep. 08 - ago. 09) En millones de DEG. Sólo Cuenta de Recursos Generales (CRG)

\begin{tabular}{|l|c|c|c|c|c|c|}
\hline & $\begin{array}{c}\text { Programas } \\
\text { aprobados }\end{array}$ & $\begin{array}{c}\text { Créditos } \\
\text { aprobados }\end{array}$ & Desembolsos & $\begin{array}{c}\text { Total } \\
\text { de cuotas }\end{array}$ & $\begin{array}{c}\text { Créditos aprobados/ } \\
\text { cuotas }\end{array}$ & $\begin{array}{c}\text { Desembolsos/ } \\
\text { cuotas }\end{array}$ \\
\hline Jjul 97-jun 98 & 14 & 31762 & 17963 & 8575 & $363 \%$ & $209 \%$ \\
\hline Sep 08-ago 09 & 20 & 105697 & 26378 & 12298 & $859 \%$ & $214 \%$ \\
\hline del cual SBA & 17 & 53513 & 26378 & 7002 & $764 \%$ & $376 \%$ \\
\hline del cual FCL & 3 & 52184 & 0 & 5296 & $985 \%$ & $0 \%$ \\
\hline
\end{tabular}

Total de cuotas: total de cuotas de los países que suscribieron un acuerdo.

Fuente: Elaboración propia con base en www.imf.org

Al comparar la proporción de los créditos con las cuotas de los países solicitantes, se observa que la relación con lo comprometido se ha incrementado en casi una vez y media, y que la relación entre los desembolsos y las cuotas -si se toman sólo los SBA- creció más de 75\% en relación con 1997-1998. La introducción de la LCF ha ampliado ostensiblemente los límites de acceso, lo cual se verifica en el aumento de la magnitud del crédito autorizado por el FMI. Por lo tanto, puede concluirse que ya sea en términos absolutos como en relación con las cuotas de los países, el Fondo ha incrementado su financiamiento.

\subsection{Límites de acceso y características}

La reforma también procuró aumentar el financiamiento mediante la duplicación de los límites de acceso normales. El cuadro 4 presenta la distribución de los acuerdos de financiamiento respecto del tipo de acceso autorizado (excepcional o no). De los

8 Se entiende por crisis financiera el proceso comprendido por las fases de agotamiento del auge económico, depresión, revulsión y pánico. Esta última se caracteriza por la conversión de activos inmobiliarios o financieros a dinero o reembolso de la deuda, acompañado de un crack en los precios de los bienes, inmuebles, acciones, bonos, o lo que sea que haya constituido el objeto de la manía en el auge (Kindleberger, 1991: 21).

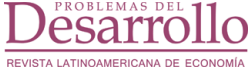


once acuerdos aprobados antes de la reforma (entre septiembre de 2008 y marzo de 2009, inclusive), sólo uno fue de acceso normal; pero luego de la reforma, de seis acuerdos aprobados, cinco fueron de acceso normal. De los datos se infiere que menos que posibilitar un aumento, la duplicación de los límites de acceso legitimó una situación que se estaba dando de hecho desde el inicio de la crisis, consecuencia de la alta necesidad de financiamiento de los países y la igualmente alta disponibilidad de recursos del organismo.

Cuadro 4

F MI: Crédito aprobado por tipo de acc eso, sep. 08 - ago. 09 Sólo CRG

\begin{tabular}{|l|c|c|}
\hline & Acceso excepcional & Acceso normal \\
\hline Sep 08-ago 091 & 11 & 6 \\
\hline Prerreforma & 10 & 1 \\
\hline Posrreforma & 1 & 5 \\
\hline
\end{tabular}

${ }^{1}$ Excluye créditos LCF.

Fuente: Elaboración propia con base en www.imf.org

La reforma también planteó la posibilidad de permitir, si fuera necesaria, una mayor concentración de los desembolsos al inicio del programa. Los datos del cuadro 5 presentan el promedio de la proporción de desembolsos realizados al inicio del acuerdo sobre el total del monto original autorizado. Se elige hacer un promedio ya que aquí interesa dar cuenta del comportamiento del FMI frente a cada acuerdo?

Cuadro 5

FMI: Promedio de la proporción primer desembolso sobre crédito total aprobado (jul. 97 - jun. 98 y sep. 08 - ago. 09). Excluye acuerdos precautorios

\begin{tabular}{|c|c|}
\hline & SBA \\
\hline Jul 97 - jun 98 & $29 \%$ \\
\hline Sep 08- ago 09 & $35 \%$ \\
\hline Prerreforma & $39 \%$ \\
\hline Posrreforma & $26 \%$ \\
\hline
\end{tabular}

Fuente: Elaboración propia con base en www.imf.org.

9 Si se tomara la proporción agregada -esto es, sumando todos los valores-, se correría el riesgo de sobreestimar el resultado al imputar a otros acuerdos magnitudes que refieren sólo a uno de ellos. Por ejemplo, el Fondo otorgó en 1997 grandes desembolsos a Tailandia y Corea del Sur-que implicaron $41 \%$ y $30 \%$ del monto original comprometido, respectivamente-, pero al compararlo con el resto de los casos de ese periodo se verifica que se trató de una decisión excepcional. Asimismo, se consideran sólo los acuerdos que incluyeron desembolsos efectivos, a fin de evitar la subestimación del resultado. 
El promedio por acuerdo Stand-By entre desembolsos iniciales sobre montos acordados subió de $29 \%$-en los acuerdos otorgados durante el primer año de la crisis del Sudeste asiático- a 35\%. Esto significa que, en promedio, el primer desembolso que otorgó el FMI al inicio de cada acuerdo durante la crisis de 1997-1998 equivalía a 29\% del total aprobado. Mientras que en la actualidad ese valor asciende a 35\%, esto es, si el Fondo aprueba una línea de crédito por DEG 100, su primer desembolso asciende a DEG 35 en promedio. Si bien hubo crecimiento, la magnitud del mismo no manifiesta un cambio sobresaliente en este punto.

No obstante, si se comparan los datos relativos a los periodos previo y posterior a la reforma, el promedio de desembolsos en relación con lo acordado en lugar de aumentar, disminuye. Mientras que en promedio en cada acuerdo aprobado antes de la reforma se desembolsaba 39\% del crédito comprometido, ese valor cae al 26\% para el periodo posterior a la misma.

Es posible arriesgar una explicación tentativa al hecho de que la reforma no haya generado cambios sobre este último punto. La propuesta de permitir -cuando sea necesario- una mayor concentración de los desembolsos al inicio del acuerdo formaba parte, junto con la de reducir la frecuencia de las revisiones, del intento de flexibilizar el uso de los SBA, por medio de la disminución de la periodicidad de la intervención del Fondo en los países.

No obstante, para que hubiera una mayor concentración del acceso al inicio debería existir una necesidad real del país basada en el desequilibrio de su balanza de pagos, el entorno económico externo, el acceso a otras fuentes de financiamiento y la conveniencia de mantener un nivel apropiado de reservas (IMF, 2009d). En este sentido, el hecho de que la proporción de desembolsos iniciales sobre el total del crédito aprobado fuera mayor antes que después de la reforma, podría ser reflejo no de una limitación inherente a la reforma, sino de una disminución de la necesidad relativa de los países prestatarios de acceso a financiamiento rápido y de gran magnitud. Esto se entendería por dos motivos, interrelacionados entre sí: 1) los países que recibieron desembolsos antes de la reforma fueron más afectados por la crisis que aquellos que recibieron desembolsos con posterioridad a la misma (mientras que los primeros sufrieron -en promedio- una caída del PIB real en 2009 de 7.38\%, los segundos cayeron "sólo" un 1.74\% ${ }^{10}$ ); 2) la aprobación de la reforma coincidió con el inicio de una lenta estabilización del sistema

10 Elaborado con base en estimaciones del FMI (www.imf.org), disponibles en columna P del cuadro A.2.

\section{Désarrollo}


financiero internacional, luego del momento más crítico en marzo de $2009^{11}$. Podría pensarse, entonces, que la relativa -aunque frágil- mejoría de las condiciones económicas a escala global, sumada a que la mayor parte de los países más afectados ya había suscrito créditos, explica la disminución de los desembolsos iniciales en los acuerdos del FMI. Con todo, es prudente esperar un lapso mayor antes de establecer un juicio concluyente sobre el alcance de la reforma en este punto.

\section{Evidencia empírica acerca de la política de condicionalidad}

\subsection{Una condicionalidad más enfocada}

La incidencia de la reforma sobre la política de condicionalidad presenta matices y un alcance desigual sobre los diferentes aspectos que la componen. Esto obliga a un análisis pormenorizado, del cual aquí se presenta una primera aproximación que se enfoca en el comportamiento que han seguido las variables centrales ${ }^{12}$.

Como es sabido, existen dos tipos de condicionalidades: cuantitativas y estructurales. Las primeras -por cierto, las más tradicionales en cuanto a su aplicación- refieren a metas numéricas sobre indicadores macroeconómicos como saldo fiscal, endeudamiento y base monetaria. Las segundas consisten en reformas del marco institucional de la economía. Aquí la variedad es mayor y se cuentan, entre otras, privatizaciones, desregulaciones y reformas al régimen financiero, tributario o laboral. Respecto de las estructurales, la reforma señaló la necesidad de profundizar el enfoque de las mismas.

En el cuadro 6 se observa que el Fondo avanzó en el enfoque hacia sus áreas de responsabilidad principal ${ }^{13}$. En la etapa posterior a la reforma, las áreas de política

11 En medio del pánico financiero provocado por las dudas que existían respecto de la voluntad del gobierno norteamericano para intervenir y evitar la quiebra de corporaciones como AIG, Citibank o Bank of America, la cotización del índice SP500 -que agrupa a las 500 empresas más grandes del mundo- se había derrumbado a niveles similares a los de 1996.

12 Para obtener los datos correspondientes al periodo septiembre 2008-agosto 2009, se analizaron todas las condicionalidades formales incluidas en los Staff Reports publicados durante ese periodo, correspondientes a los acuerdos indicados en el cuadro A.3. De este modo, se conformó una base de datos que contiene 102 condicionalidades cuantitativas y 170 estructurales, incluidas en 16 acuerdos SBA y sus revisiones aprobadas en el periodo indicado (de los 20 acuerdos aprobados, no hay datos sobre el de Sri Lanka ni, por supuesto, sobre los tres LCF que no incluyen condicionalidades). Aunque para alcanzar un resultado más completo sería aconsejable considerar, también, las medidas que sólo son mencionadas en el cuerpo de Memorandum de política económica de los acuerdos, debido a las restricciones de espacio y a que se priorizó la posibilidad de ofrecer resultados -aunque sea parciales-que permitan responder el interrogante establecido. El análisis de esos datos se dejó para una próxima etapa.

13 Para obtener la distribución por área, se relevaron todas las condicionalidades y se las discriminó por área según el objetivo perseguido. Luego se las distribuyó según el mes en el que fue 
Cuadro 6

FMI: Condicionalidad estructural. Distribución por área (sep. 08 - ago. 09)

\% de todas las condiciones. Sólo CRG

\begin{tabular}{|l|c|c|}
\hline & Prerreforma sep. 08 - mar. 09 & Posrreforma abr. 09 - ago. 09 \\
\hline Sector financiero & $42 \%$ & $34 \%$ \\
\hline Política cambiaria y monetaria & $27 \%$ & $25 \%$ \\
\hline Gasto público & $27 \%$ & $27 \%$ \\
\hline Políitca fiscal & $6 \%$ & $15 \%$ \\
\hline Resto & $10 \%$ & $5 \%$ \\
\hline
\end{tabular}

Fuente: Elaboración propia con base en IMF Staff Reports.

financiera, monetaria, cambiaria y fiscal concentran $95 \%$ de las condiciones estructurales. Como era de esperarse, en la primera etapa del periodo actual se observa una alta proporción de condiciones orientadas hacia el sector financiero, habida cuenta de la necesidad de aplicar medidas que estabilizaran al sector de la economía más afectado por la crisis.

Sin embargo, cabe señalar que menos que introducir un cambio estructural en este punto, la reforma consolidó y profundizó un proceso iniciado antes de su aprobación. Esto se verifica en que durante el periodo 1995-2000, las cuatro áreas principales del Fondo concentraron 51\% del total de condicionalidades estructurales; y durante 20012004, ese número ascendió a 64\% (OEI-FMI, 2007: 5).

La "especialización" del Fondo en sus áreas centrales marca un contraste con la dispersión que mostraban sus exigencias en la década de 1990, cuando, siguiendo el decálogo del Consenso de Washington, se extendían a otras áreas como privatizaciones, desregulaciones y reformas educativas y laborales. Otra cuestión a destacar es que los acuerdos con Pakistán, Armenia y Mongolia incluyeron medidas dirigidas al fortalecimiento de las redes de protección social (en los tres casos se trató de parámetros de referencia). Esto último podría interpretarse como una respuesta al planteamiento del G20 acerca de minimizar el impacto social negativo de la recesión (G20, 2008b). Aunque con tibieza, el Fondo parecería haber mejorado algunas prácticas criticadas en el pasado, referidas a la intervención en áreas ajenas a su expertise, y a la mitigación de los efectos adversos de los programas sobre los sectores más

aprobado el acuerdo o la revisión en la que están incluidas. Así se consolidaron dos conjuntos de condiciones por área, uno relativo a los meses previos a la reforma, y otro a los meses posteriores. De esos dos conjuntos se calculó el porcentaje para cada área sobre el total de las condiciones. La clasificación de las áreas en las que se distribuyen las condiciones estructurales se basa en OEI-IMF (2007: 25). 
vulnerables. Con todo, debe señalarse que la disposición del FMI de establecer protecciones sobre los sectores vulnerables, se lleva a cabo mediante la imposición de condicionalidades que, irónicamente, exceden su área de expertise.

\subsection{Condicionalidad estructural por área, tipo y grado de exigencia}

Buscando reducir el estigma que establecían las condicionalidades sobre los prestatarios, la reforma planteó la intención de reducir las tradicionales condicionalidades ex post y apoyarse más en los criterios de precalificación. La introducción de la LCF avanzó en este sentido, ya que consiste en una línea de crédito que no incluye condiciones ex post, y se otorga a partir de la consideración de la robustez de las variables macroeconómicas ${ }^{14}$. Además se interrumpió el uso de los criterios de ejecución estructurales, los cuales son condiciones específicas que deben ser cumplidas antes de una fecha determinada para evitar la suspensión del programa y, por ende, de los desembolsos -aunque el Fondo puede otorgar una dispensa (waiver) si considera que el país ha adoptado o planea adoptar medidas para corregir la desviación. De este modo, los acuerdos relacionados con las condiciones estructurales ahora incluyen exclusivamente acciones previas y parámetros de referencia. Las primeras son las más duras ya que imponen exigencias que deben ser cumplidas antes de la aprobación del acuerdo o la revisión, mientras que las segundas son las más blandas, porque su evaluación se realiza en la instancia de revisión (no imponen fechas límite adicionales a la revisión) y su incumplimiento no amerita sanción en términos formales (Woods, 2006: 375).

Resulta interesante analizar la evolución de la distribución de las reformas estructurales por grado de exigencia luego de la eliminación de los criterios de ejecución estructurales, a fin de observar si aumentaron en mayor proporción los parámetros de referencia o las acciones previas. En el cuadro $8^{15}$ se observa que, luego de la eliminación, la proporción correspondiente a ellas se distribuyó entre ambos tipos de condiciones, siendo mayor el aumento de los parámetros de referencia. Así, por cada 10 condiciones que el organismo exige en cada nuevo acuerdo o revisión luego de la reforma, poco más de siete son parámetros de referencia y poco menos de tres son acciones previas.

14 La redacción de los Staff Reports correspondientes a la aprobación de los LCF en México, Polonia y Colombia manifiesta esta característica. En dos de ellos -Polonia y Colombia- el primer subtítulo hace referencia expresa a los "sólidos fundamentos" en los que se apoya la economía, mientras en el restante, el primer cuadro se titula "sólido desempeño", refiriéndose a la evolución en los últimos años (IMF, 2009e: 3; 2009f: 3; 2009g: 4).

15 Elaborado con un criterio similar al del cuadro 6, sólo que las condiciones se discriminaron por tipo, no por área. 
Cuadro 7

Características de las condicionalidades del FMI, según tipo y grado de exigencia

\begin{tabular}{|c|c|l|}
\hline Cuantitativas & Estructurales & \multicolumn{1}{c|}{ Características } \\
\hline Acciones previas & Acciones previas & $\begin{array}{l}\text { - Plazo de cumplimiento: antes del inicio de las negociaciones } \\
\text {-Sanción ante incumplimiento: suspensión inmediata de las negociaciones }\end{array}$ \\
\hline Criterios de ejecución & Criterios de ejecución & $\begin{array}{l}\text { - Plazo de cumplimiento: variable (puede ser antes de la revisión o antes) } \\
\text {-Sanción ante incumplimiento: suspensión del programa } \\
\text { (aunque puede perdonarse) }\end{array}$ \\
\hline Metas indicativas & Parámetros de referencia & $\begin{array}{l}\text { - Plazo de cumplimiento: en la revisión } \\
\text {-Sanción ante incumplimiento: formalmente ninguna }\end{array}$ \\
\hline
\end{tabular}

${ }^{1}$ Eliminados en la reforma de fines de marzo de 2009.

Fuente: Elaboración propia con base en FMI.

Cuadro 8

F MI: Promedio de condicionalidades estructural es por grado de exigencia, sep. 08 - ago. 09 \% de todas las condiciones. Sólo CRG

\begin{tabular}{|l|c|c|}
\hline & Prerreforma & Posrreforma \\
\hline Acciones previas & $23 \%$ & $27.5 \%$ \\
\hline Criterios de ejecución & $19 \%$ & $0.0 \%$ \\
\hline Parámetros de referencia & $58 \%$ & $72.5 \%$ \\
\hline
\end{tabular}

Fuente: Elaboración propia con base en FMI.

El aumento proporcional de los parámetros de referencia en comparación con otros años -la proporción por año de acuerdo durante 1998 fue de aproximadamente 48\% (OEI-FMI, 2007: 19)- permite pensar que las revisiones cobrarán aún más importancia como instancia de negociación, ya que constituirán la fecha límite para el cumplimiento de las reformas estructurales exigidas. $\mathrm{Al}$ mismo tiempo, dado que no existen criterios claramente establecidos respecto de la continuidad de un programa ante el incumplimiento de estas metas, es posible que esto aumente el poder de negociación de los técnicos y la gerencia del organismo, en tanto su criterio subjetivo tendrá mayor peso a la hora de la aprobación de revisiones.

Aunque hasta aquí hemos visto que la reforma ha incorporado algunas novedades, autores como Weisbrot, Cordero y Sandoval (2009) y Weissmann (2009) coinciden en señalar que el Fondo sigue exigiendo medidas de política económica de orientación ortodoxa, que limitan la posibilidad de implementar políticas contracíclicas y profundizan el ciclo recesivo. A fin de realizar un análisis que permita observar si ha habido cambios en la orientación política de las exigencias, se comparó la distribución de condiciones estructurales combinando los criterios de área y grado de 
exigencia. Se considera que las áreas con mayor proporción de condiciones de acción previa son las que revisten más importancia para el Fondo, ya que es donde demuestra menos tolerancia. Por ejemplo, si una condición relativa al establecimiento de redes de protección social es un parámetro de referencia, pero otra que exige un recorte en el gasto corriente es acción previa, se interpreta que el organismo pone más énfasis sobre la segunda medida. Por supuesto, sin dejar de considerar la importancia de la primera.

\section{Gráfica 1}

FMI: distribución de condicionalidades estructurales por área y grado de exigencia; antes y después de la reforma. Todas las condicionalidades. Sólo CRG

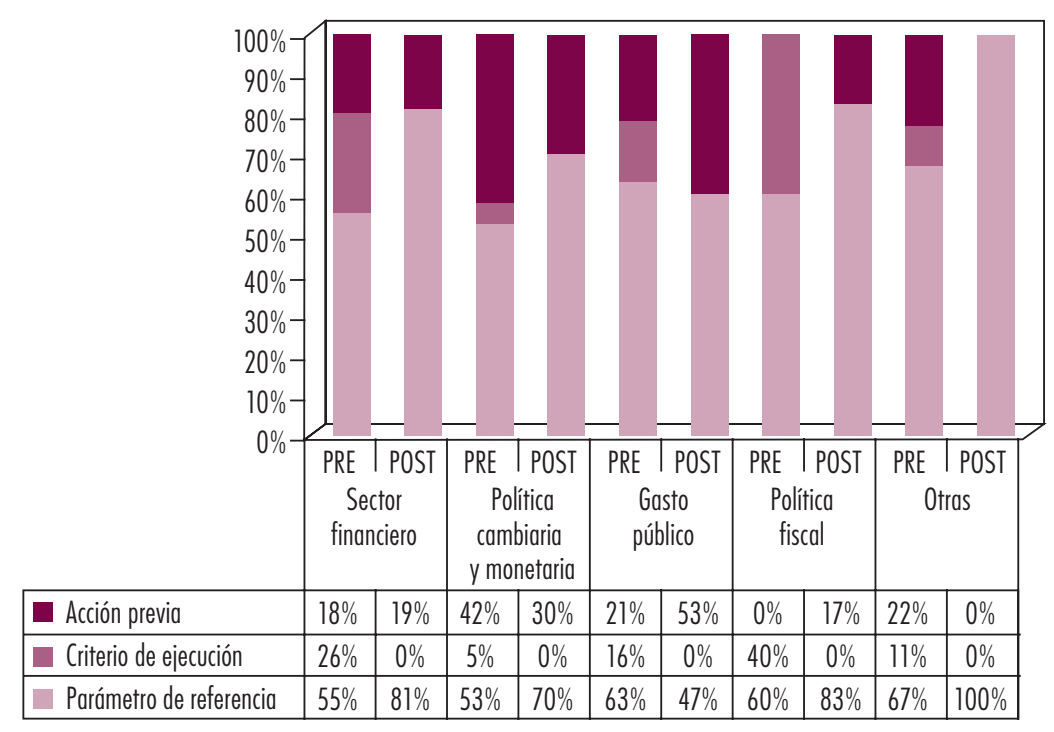

Fuente: Elaboración propia con base en cuadro A.5. (Datos del FMI.)

La gráfica 1 permite observar que la reforma introdujo un cambio en la composición cualitativa de las condiciones según la relación de área y grado de exigencia. Esto es, al eliminarse los criterios estructurales de ejecución, esas condiciones no se distribuyeron equitativamente entre las áreas. El área donde más aumentan las exigencias en términos de proporción de acciones previas sobre el total de condiciones de esa misma área es en administración del gasto público, donde las acciones previas pasan de $21 \%$ a $53 \%$ del total. En esta área se concentran exigencias como recortes en el gasto o la aprobación de presupuestos que incluyan ajustes o limitaciones al endeudamiento, típicas de un programa de estabilización ortodoxo centrado en la búsqueda de equilibrio fiscal. Otra área que aumenta en términos relativos su proporción de metas exigentes es política 
fiscal, que pasa de no tener ninguna acción previa a poseer $17 \%$ del total. En el resto de las condicionalidades - en línea con el mayor aumento relativo de los parámetros de referencia en detrimento de las acciones previas luego de la eliminación de los criterios de ejecución-aumenta en mayor medida la proporción de parámetros de referencia.

Gráfica 2

FMI: distribución de condicionalidades estructurales por grado de exigencia y área; antes y después de la reforma. Todas las condicionalidades. Sólo CRG

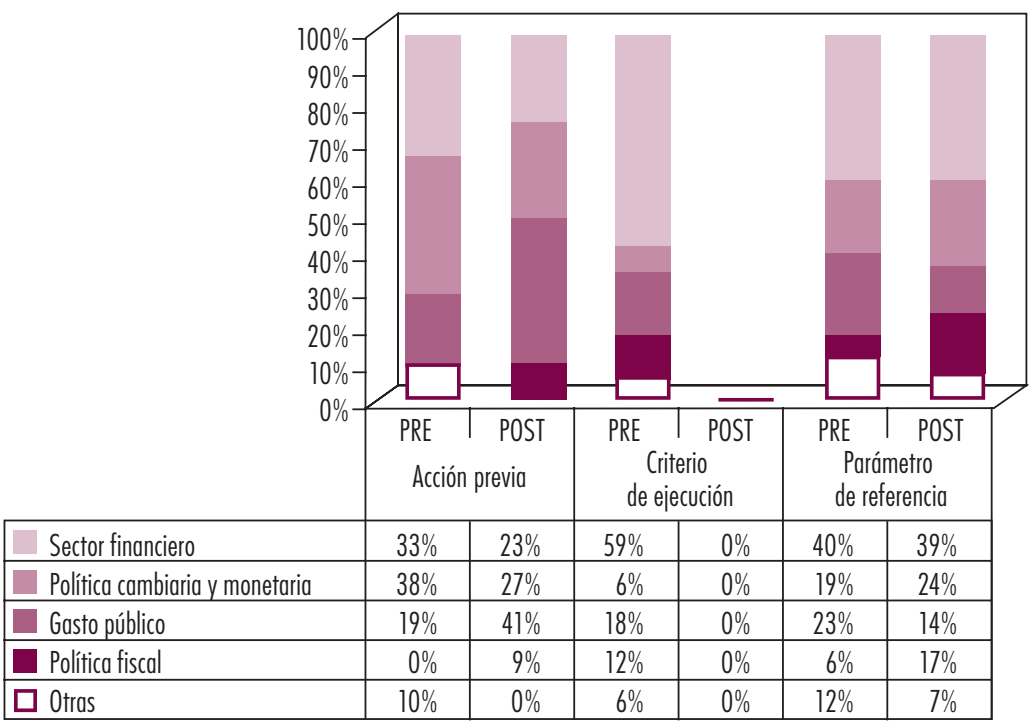

Fuente: Elaboración propia con base en cuadro A.5. (Datos del FMI.)

Tal como se desprende de la gráfica 2, las áreas en las que se concentra mayor cantidad de acciones previas luego de la reforma son administración del gasto público y política cambiaria y monetaria. Así, condicionalidades que buscan alcanzar la estabilización mediante políticas de restricción de la demanda como reducción del déficit (Ucrania y Rumania), recortes en los salarios públicos (Serbia y Bosnia) y alza de la tasa de interés local (Islandia, Pakistán y Bielorrusia -los dos primeros antes de la reforma-), son las exigidas en mayor cantidad bajo la forma de acciones previas.

\subsection{Condicionalidades cuantitativas}

Las condiciones cuantitativas también parecen reproducir esta orientación. La gráfica 3 presenta una distribución de las mismas según su objetivo y el número promedio 
por programa ${ }^{16}$. Todos los acuerdos en promedio incluyen condiciones que limitan la posibilidad de suspender -aunque sea temporalmente- el servicio de la deuda e imponen límites al endeudamiento público. Si bien es comprensible que el FMI demuestre preocupación por el mantenimiento de un nivel de endeudamiento razonable, es deseable relajar esas exigencias en contextos recesivos, ya que el uso del crédito puede contribuir a la reactivación. En ese sentido, casi todos los acuerdos en promedio imponen límites al déficit fiscal. Por ejemplo, la exigencia de implementar una ley de déficit cero en plena crisis económica, ha reaparecido en los acuerdos con Ucrania y Bielorrusia.

\section{Gráfica 3}

FMI: Condicionalidades cuantitativas por objetivo Al inicio de cada acuerdo, 16 acuerdos SBA, sep. 08 - ago. 09

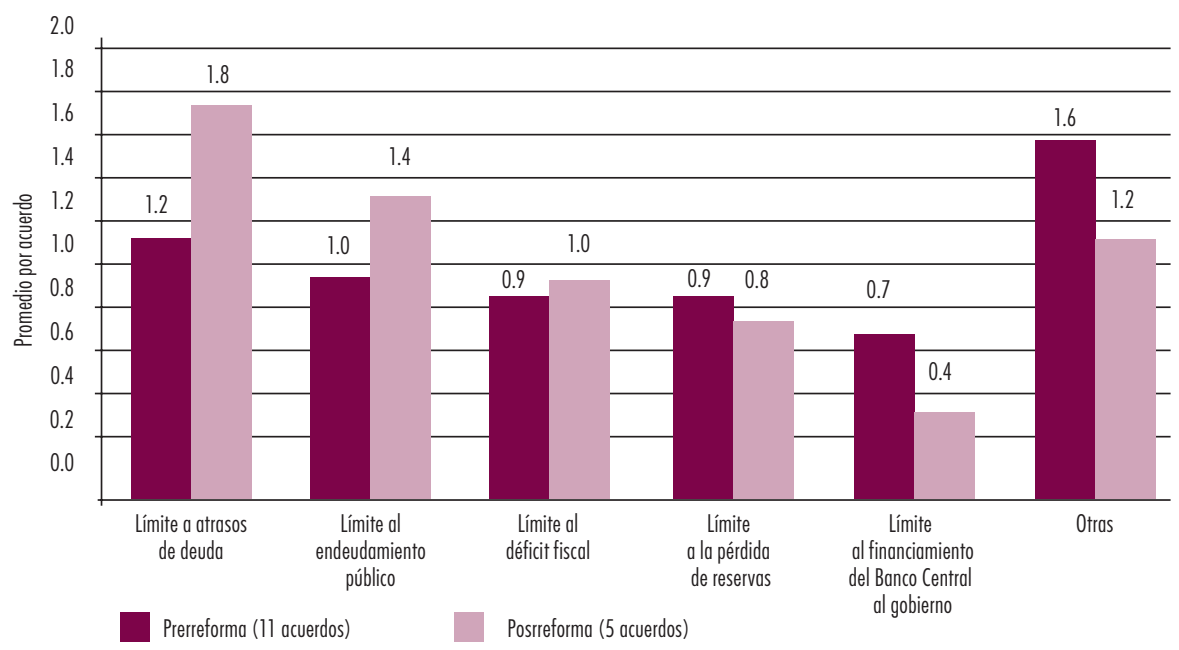

Fuente: Elaboración propia con base en IMF Staff Reports (véase cuadro A.3).

La gráfica ratifica lo señalado respecto de la concentración de las condiciones del Fondo. Luego de la reforma, disminuye el promedio por programa de condiciones incluidas en la categoría otras (por ejemplo, reforma del empleo público -Letonia, Serbia, Rumania- o mejoramiento de la gobernanza-Seychelles-), al tiempo que aumentan en proporción las primeras tres -ejes centrales de cualquier programa

16 Para la elaboración de esta gráfica se tomaron las 102 condicionalidades cuantitativas de la base de datos indicada en la nota al pie 12, y se les ordenó según fueron establecidas en acuerdos aprobados antes o después de la reforma. Luego se dividió la cantidad de condiciones por la cantidad de acuerdos, y se obtuvo un promedio por programa. Las categorías utilizadas se basan en Stone (2008: 18). 
ortodoxo-, que en tiempos de caída de la actividad, busca restablecer el equilibrio a través de la reducción de la demanda.

Cabe señalar que el establecimiento de límites al endeudamiento y al déficit fiscal eran exigencias contrarias a la posición pública del G20, que a través del financiamiento multilateral buscaba alentar en los países en desarrollo la implementación de políticas contracíclicas como el aumento del gasto público (G20, 2009a). Respecto de la exigencia de limitar los atrasos de deuda, la contradicción es menos evidente, dado que al tiempo que el G20 buscaba garantizar el acceso a financiamiento para estos países, también procuraba alcanzar la estabilización del sistema financiero internacional en el marco del respeto al paradigma del libre mercado (G20, 2009b).

Con todo, en algunos casos el Fondo aceptó la utilización de la política fiscal como instrumento para reactivar la demanda. Pero el alcance se restringe a economías pequeñas que -según la interpretación del organismo-demostraron durante los años previos un nivel de ahorro elevado (Costa Rica y Guatemala). Dado que en otros casos, como Rumania o Bosnia, las recomendaciones siguen la orientación ortodoxa, el alcance de la novedad que mostraría en relación con sus exigencias en política fiscal se relativiza, al tiempo que sugiere esperar un lapso mayor antes de establecer un juicio concluyente acerca de la existencia (y el grado) de una modificación en este punto.

\section{Perspectivas para América Latina}

En este apartado se propone una breve reflexión sobre las consecuencias de la reforma en el FMI en su relación con los países de América Latina. Para ello, es ilustrativo retomar la comparación con los países del Sudeste asiático efectuada al inicio del trabajo.

Pocos episodios motivaron críticas tan extendidas sobre el FMI como su intervención en el Sudeste asiático durante la crisis de 1997-1998. El Fondo impidió establecer controles para limitar la masiva salida de capital y exigió la implementación de un drástico ajuste a fin de lograr la estabilización. Esto profundizó la recesión, y agravó el impacto sobre los sectores menos favorecidos. La experiencia motivó que los países de la región desarrollaran instrumentos para evitar recurrir al FMI ante futuras crisis. En ese sentido, avanzaron en dos iniciativas, relacionadas entre sí: primero, aprovechando el ciclo externo favorable, aumentaron progresivamente sus reservas para disponer de un "colchón" que amortiguara las presiones que impone una crisis sobre el frente cambiario; segundo, establecieron un mecanismo de financiamiento regional que puede poner a disposición hasta US $\$ 120000$ millones (Choonsik, 28/12/09). Se trata de la Iniciativa Chiang Mai, que involucra a China, Corea del Sur, Japón -éstos aportan 80\% del capital total- y a los países integrantes de la Asociación de Naciones del Sudeste Asiático 
$(\mathrm{ASEAN})^{17}$. La disponibilidad de financiamiento regional de tal magnitud evita recurrir a los desembolsos del FMI, que en la forma de acuerdos Stand-By siguen incluyendo restrictivas condicionalidades.

Gráfica 4

FMI: acuerdos aprobados durante julio 1997-junio 1998 y sep. 2008-agosto 2009 Sólo acuerdos LCF, SAF y SBA

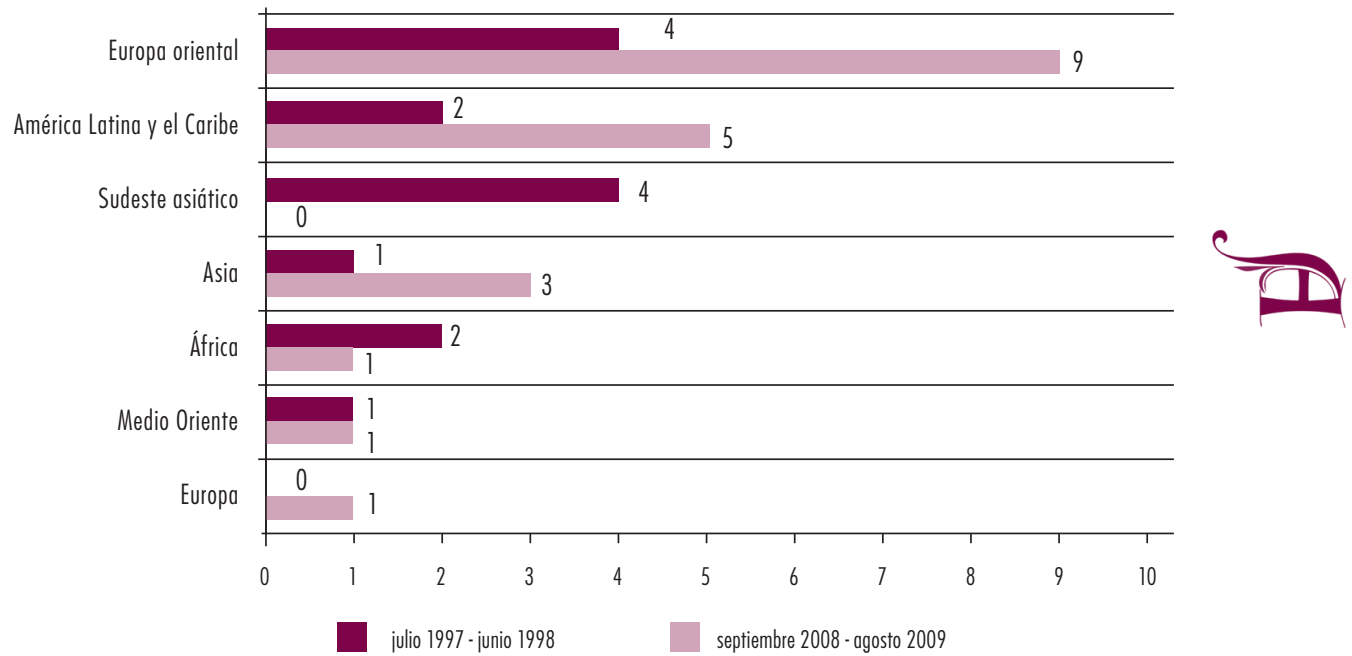

Fuente: Elaboración propia con base en www.imf.org

La gráfica 4 permite observar el resultado exitoso de esa política en relación con el objetivo de evitar recurrir al Fondo, ya que a un año del estallido de la crisis actual, ningún país del Sudeste asiático había solicitado su asistencia. Por el contrario, cinco países de América Latina habían suscrito programas. Notablemente, en todos los casos se trata de acuerdos precautorios, donde el Fondo aprueba una línea de crédito, pero los países manifiestan su compromiso de no utilizarla y reservarla para una situación de extrema necesidad. Si consideramos que en nuestra región otros países han manifestado en reiteradas ocasiones la decisión de evitar recurrir a los programas del organismo en tanto no se observen cambios de fondo en la orientación de sus recomendaciones, cabe preguntarse cómo se explica la actual situación.

En términos estilizados, pueden distinguirse tres grupos de países respecto de su relación con el Fondo durante este periodo. El primer grupo lo integran México y Colombia. Para éstos -que han accedido a la nueva LCF- podría pensarse que la

17 Países integrantes de ASEAN: Brunei, Camboya, Indonesia, Laos, Malasia, Myanmar, Filipinas, Singapur, Tailandia y Vietnam. 
situación resulta favorable, ya que obtienen apoyo financiero sin condicionalidades que les obliguen a modificar su programa económico. En el segundo grupo aparecen El Salvador, Costa Rica y Guatemala, quienes suscribieron programas condicionales con el Fondo. En estos casos -en especial en los dos últimos, posteriores a la reforma- uno de los aspectos a destacar y que permiten comprender la inclinación a suscribir un acuerdo -además del interés por acceder al financiamiento- lo constituye la disposición del Fondo a aceptar la implementación de moderadas políticas fiscales contracíclicas. En el tercer grupo se encuentran los países del Mercosur, además de Venezuela, Ecuador y Bolivia. Con algunas diferencias entre sí, este grupo parece dispuesto a evitar recurrir al financiamiento del organismo mientras implique la aceptación de condicionalidades, y en tanto, avanza en un doble sentido: por un lado, reclama la profundización de la redistribución de votos al interior del organismo a favor de los países en desarrollo; por el otro, consolida el Banco del Sur, que pondrá a disposición financiamiento por US \$20 000 millones a los países miembro (Piqué, 27/09/09). Aunque con un monto más limitado, el Banco ofrecerá una fuente de financiamiento alternativa al FMI, buscando un efecto similar al conseguido por la Iniciativa Chiang $\mathrm{Mai}^{18} \mathrm{y}$, por ende, ofreciendo una posibilidad de aumentar la autonomía relativa de los países de la región frente al organismo.

\section{Reflexiones finales}

A pesar de que ha transcurrido un periodo breve desde la reforma, la presentación de los datos principales sobre la política financiera y de condicionalidad del FMI permite establecer unas primeras observaciones en relación con el alcance de la misma. Aunque, huelga decir, el Fondo no propuso expresamente modificar la orientación de política económica en sus recomendaciones, algunos indicios sugieren que podría haber modificaciones en este sentido. Por una parte, la referencia a que los programas estén hechos adecuadamente a la medida de la fortaleza de las políticas y los fundamentals de los países. Y por otra, las reiteradas afirmaciones de varios de sus funcionarios -entre ellos el director gerente- subrayando la necesidad de que los países posean márgenes de acción fiscal y monetaria para implementar programas de estímulo que impulsen la demanda y el consumo (Muchhala, 2009: 2).

Sin embargo, el FMI presenta, luego de la reforma, aspectos novedosos junto a otros

18 Cabe señalar que mientras la Iniciativa Chiang Mai consiste en una red constituida por acuerdos tipo swap de intercambio de monedas entre los bancos centrales de los países que la conforman, el Banco del Sur administrará un capital aportado por los países que lo integran. 
de continuidad. En relación con los primeros se cuentan los préstamos de mayor magnitud, la puesta a disposición -mediante la LCF- de alto financiamiento sin condicionalidades ex post (aunque sí exigentes criterios de precalificación), la profundización de la concentración de la condicionalidad estructural en sus áreas centrales, y cierto interés por la protección social de los sectores más vulnerables. No obstante, la orientación de sus recomendaciones de política muestra continuidades en aspectos esenciales. En especial, la no aceptación de controles cambiarios o de capitales -aunque sean temporales-, junto con un mayor énfasis en las condiciones estructurales referidas a la disminución del gasto público. Aunque en algunos acuerdos el Fondo aceptó una elevación del déficit para reactivar la demanda, los datos agregados indican un aumento del énfasis en las condiciones referidas a este punto. Esto lleva a que sus condiciones, aunque limitadas a pocas áreas, reproduzcan en su contenido el esquema de ajuste con libre movilidad de capitales, que puede profundizar el impacto del shock externo.

El análisis precedente pone de manifiesto que aunque ambos aspectos de la reforma muestran matices, la misma fue más efectiva en la política crediticia que en la política de condicionalidad. Esto es, mientras que el Fondo ha logrado reposicionarse en su papel de prestamista en contextos de crisis, la orientación de la política económica de sus exigencias muestra más continuidades que cambios luego de la reforma. A modo de hipótesis de trabajo para futuras investigaciones, podría argumentarse que esta situación se explica por dos factores. Primero, es probable que los países centrales -que ostentan una posición dominante sobre el organismo- se mostraran más interesados en que el Fondo aumentara su financiamiento que en la reorientación intelectual de sus condicionalidades. En muchas ocasiones, los créditos del Fondo fueron motivados, más que por la voluntad del organismo de morigerar el impacto de las crisis, por el interés de los países industrializados de evitar pérdidas a las filiales de sus corporaciones multinacionales en las economías emergentes, y el antecedente de Islandia -entre otros- habilita a pensar que esta crisis no plantearía una excepción en este sentido. Segundo, a pesar de existir un consenso entre economías industrializadas y emergentes respecto de la necesidad de que el financiamiento del Fondo alentara la implementación de políticas contracíclicas en estas últimas, no deben soslayarse las resistencias que podría encontrar esa propuesta en el paradigma intelectual que orienta los análisis de los técnicos del organismo.

Lo antedicho permite sugerir dos recomendaciones sobre el sentido que deberían tomar las reformas futuras en el organismo. Primero, lograr la tan discutida revisión de las cuotas que permita una distribución más equilibrada de poder dentro del organismo, favoreciendo a las economías emergentes. Segundo, reexaminar los criterios, supuestos y análisis económicos que los técnicos del Fondo utilizan para elaborar recomendaciones de política.

Vol. 41, núm. 160, enero-marzo / 2010

Desares roullo 
Arceo, Enrique, Claudio Golonbek y Romina Kupelian, "Crisis mundial. Elementos para su análisis", Documentos de Trabajo, núm. 26, CEFID-AR, Buenos Aires, julio de 2009.

Choonsik, Yoo, "Este asiático lanzará fondo emergencia de 120000 mln dlrs marzo", Reuters, 28 de diciembre de 2009: http:// lta.reuters.com/articlePrint?articleId=LTA SIE5BR06220091228.

G20, "Declaration on delivering resources through the international financial institutions", Londres, 2 de abril de 2009a: http:// www.g20.utoronto.ca/2009/2009delivery. html.

"G20 leaders statement: The Pittsburgh summit", Pittsburgh, 24-25 de septiembre de 2009b: http://www.g20.utoronto.ca/2009/2009communique0925.html.

, "Declaration of the summit on financial markets and the world economy", Washington DC, 15 de noviembre de 2008a: http://www.g20.utoronto. $\mathrm{ca} / 2008 / 2008$ declaration $1115 . \mathrm{html}$.

, "Communiqué of the meeting of the G20 finance ministers and Central Bank governors", Sao Paulo, 9 de noviembre de 2008b: http://www.g20.utoronto. $\mathrm{ca} / 2008 / 2008$ communique1109.html.

Griesgraber, Jo Marie, "Reforms for major new roles of the International Monetary Fund? The IMF Post-G-20 summit", Global Governance, vol. 15, núm. 2, 2009, pp.179185.

Independent Evaluation Office IEO-IMF, "Structural conditionality in IMF-supported programs. Background documents", 21 de octubre de 2007: http://www.ieo-imf.org/ eval/complete/pdf/01032008/SC_background_documents.pdf.

IMF, "IMF overhauls nonconcessional lending facilities and conditionality", Public Information Notice, núm. 09/ 40, 3 de abril de 2009a: http://www.imf.org/external/np/sec/ pn/2009/pn0940.htm.

"Bolstering the IMF's lending capacity", 2009b: http://www.imf.org/external/ $\mathrm{np} / \mathrm{exr} / \mathrm{faq} / \mathrm{contribution}$.htm, consultado: 22/12/09.

"IMF executive board approves limited sales of gold to finance the Fund's new income model and to boost concessional lending capacity", Press Release, núm. 09/310, 18 de septiembre de 2009c: http:// www.imf.org/external/np/sec/pr/2009/ pr09310.htm.

"Decision 7925-(85/38), amended by Decision 14281-(09/29). Article V, Section 3 (a), (b) and (c)", Selected Decisions and Selected Documents of the IMF, Thirty-Third Issue, 2009d: http:// www.imf.org/external/pubs/ft/sd/index. asp?decision=7925-\%2885/38\% 29 .

" "Colombia: Arrangement under the flexible credit line-staff report; staff supplement; press release on the executive board discussion; and statement by the executive director for Colombia", Country Report, núm. 09/153, Washington, mayo de 2009e: http://www.imf.org/external/pubs/ $\mathrm{ft} / \mathrm{scr} / 2009 / \mathrm{cr} 09153$.pdf.

" "Republic of Poland: Arrangement under the flexible credit line-staff report; staff supplement; press release on the executive board discussion; and statement by the executive director for the Republic of Poland", Country Report, núm. 09/ 138, Washington, mayo de 2009f, http://www. imf.org/external/pubs/ft/scr/2009/cr09138. pdf.

"Mexico: Arrangement under the flexible credit line-staff report; staff supplement; and press release on the executive board discussion", Country Report, núm. 09/ 126, Washington, abril de 2009g: http:// www.imf.org/external/pubs/ft/scr/2009/ cr09126.pdf.

"IMF executive board concludes review of access policy in the credit tranches and under the extended fund facility and the poverty reduction and growth facility, and exceptional access policy", Public Information Notice, núm. 08/30, 7 de marzo de 2008a: http://www.imf.org/external/np/sec/ pn/2008/pn0830.htm.

"IMF executive board reviews the Fund's financing role", Public Information Notice, núm. 08/ 131, 9 de octubre de 2008b: http://www.imf.org/external/np/sec/ pn/2008/pn08131.htm.

Kindleberger, Charles, Manías, pánicos y cracks. Historia de las crisis financieras, Barcelona, Ariel, 1991.

Vol. 41, núm. 160, enero-marzo / 2010 
Molina, Nuria y Javier Pereira, "Critical conditions. The IMF maintains its grip on lowincome governments", Eurodad, abril de 2008: http://www.eurodad.org/uploadedFiles/Whats_New/Reports/Critical_conditions.pdf.

Muchhala, Bhumika, "The IMF: Resurgent but unchanged", Third World Resurgence, núm. 225, Penang, Third World Network, mayo de 2009: http://www.twnside.org.sg/title2/ resurgence/2009/twr225.htm.

Oficina de Evaluación Independiente OEI-FMI, "Evaluación de la OEI sobre la condicionalidad estructural de los programas respaldados por el FMI", Washington, 2007.

Piqué, Martín, "En el camino entre el FMI y el Banco del Sur", Página 12, 27 de septiembre de 2009.

Stone, Randall, "The scope of IMF conditionality", 1st Annual PEIO Conference, ETH Zurich-University of Zurich, 2008: http://www.cis.ethz.ch/events/past_events/ PEIO2008/Stone_Scope.IMF.Conditionality.

Torres, Héctor, "The idle fireman", Project Syndicate, 2008: http://www.project-syndicate. org/commentary/torres2.
, "Reforming the International Monetary Fund-Why its legitimacy is at stake", Journal of International Economics Law, vol. 10, núm. 3, 2007, pp. 443-460.

Ward, Andrew y George Parker, "IMF plays down Iceland loan rift", Financial Times, 31 de julio de 2009.

Weisbrot, Mark, José Cordero y Luis Sandoval, "Empowering the IMF: Should reform be a requirement for increasing the Fund's resources?", Washington DC, Center for Economic and Policy Research, 2009: http:// www.cepr.net/documents/publications/ imf-reform-2009-04.pdf.

Weissmann, Robert, "A new life for the IMF: Capitalising on crisis", Third World Resurgence, núm. 225, mayo de 2009: http:// www.twnside.org.sg/title2/resurgence/2009/twr225.htm.

Woods, Ngaire, "Understanding pathways through financial crises and the impact of the IMF: An introduction", Global Governance, vol. 12, núm. 4, 2006, pp. 373-393. 
Anexos

Cuadro A.1

FMI: Detalle de acuerdos aprobados, jul. 97-jun. 98

En millones de DE G. Sólo CRG

\begin{tabular}{|c|c|c|c|c|c|c|c|c|c|c|c|c|c|c|c|}
\hline$A$ & $B$ & $C$ & D & $E$ & $F$ & G & $H$ & 1 & J & $k$ & L & M & N & 0 & $P$ \\
\hline Tipo & Prec & Acceso & País & Fecha & $\begin{array}{c}\text { Duración } \\
\text { meses }\end{array}$ & $P I B$ & Cuota & $\begin{array}{c}\text { Monto } \\
\text { aprobado }\end{array}$ & $\begin{array}{c}\text { \% del } \\
P I B\end{array}$ & $\begin{array}{l}\% \text { de } \\
\text { cuota }\end{array}$ & Desembolsos & $\begin{array}{c}\text { Relación } \\
\text { L/I }\end{array}$ & $\begin{array}{c}\text { Monto } \\
\text { aprobado } \\
\text { al inicio }\end{array}$ & $\begin{array}{c}\text { Primer } \\
\text { desembolso }\end{array}$ & $\begin{array}{c}\text { Relación } \\
0 / N\end{array}$ \\
\hline SBA & & $E$ & Tailandia & ag097 & 34 & 126532 & 574 & 2900 & $2.29 \%$ & $505 \%$ & 2100 & $72 \%$ & 2900 & 1200 & $41 \%$ \\
\hline SBA & & $\mathrm{NE}$ & Ucrania & ago 97 & 12 & 31014 & 997 & 399 & $1.29 \%$ & $40 \%$ & 181 & $45 \%$ & 399 & 36 & $9 \%$ \\
\hline SBA & Prec & $N E$ & Letonia & oct 97 & 18 & 3951 & 91 & 33 & $0.84 \%$ & $36 \%$ & 0 & $0 \%$ & 33 & 0 & $0 \%$ \\
\hline SAF & & NE & Pakistán & oct97 & 36 & 53788 & 758 & 455 & $0.85 \%$ & $60 \%$ & 57 & $13 \%$ & 455 & 38 & $8 \%$ \\
\hline SAF & & $\mathrm{NE}$ & Yemen & oct 97 & 36 & 4518 & 176 & 106 & $2.35 \%$ & $60 \%$ & 9 & $8 \%$ & 106 & 9 & $8 \%$ \\
\hline SBA & & $E$ & Indonesia & nov 97 & 36 & 174376 & 1498 & 8338 & $4.78 \%$ & $557 \%$ & 2935 & $35 \%$ & 7338 & 2200 & $30 \%$ \\
\hline SBA & & $E$ & Corea & $\operatorname{dic} 97$ & 36 & 388071 & 1634 & 15500 & $3.99 \%$ & $94 \% \%$ & 12600 & $87 \%$ & 15500 & 4100 & $26 \%$ \\
\hline SAF & & NE & Panamá & $\operatorname{dic} 97$ & 36 & 6483 & 150 & 120 & $1.85 \%$ & $80 \%$ & 18 & $15 \%$ & 120 & 10 & $8 \%$ \\
\hline SBA & Prec & $N E$ & Estonia & $\operatorname{dic} 97$ & 15 & 3277 & 46 & 16 & $0.49 \%$ & $35 \%$ & 0 & $0 \%$ & 61 & 0 & $0 \%$ \\
\hline SAF & Prec & NE & Argentina & feb 98 & 36 & 189261 & 1537 & 2080 & $1.10 \%$ & $135 \%$ & 0 & $0 \%$ & 2080 & 0 & $0 \%$ \\
\hline SBA & Prec & $N E$ & Cabo Verde & feb 98 & 14 & 349 & 7 & 2 & $0.57 \%$ & $29 \%$ & 0 & 0 & 2 & 0 & $0 \%$ \\
\hline SBA & Prec & $N E$ & Filipina & abr 98 & 24 & 58674 & 633 & 1021 & $1.74 \%$ & $167 \%$ & 0 & $0 \%$ & 1021 & 0 & $0 \%$ \\
\hline SBA & & NE & Bosnia-Herzeg. & may 98 & 12 & 2268 & 121 & 61 & $2.69 \%$ & $50 \%$ & 24 & $39 \%$ & 61 & 24 & $39 \%$ \\
\hline SBA & & $N E$ & Zimbawe & jun 98 & 13 & 6091 & 353 & 131 & $2.15 \%$ & $37 \%$ & 39 & $30 \%$ & 131 & 39 & $30 \%$ \\
\hline
\end{tabular}

Prec: Acuerdo precautorio

Acceso: Excepcional (E) o normal (N)

PIB: valores a finales de 1996. PIB a dólares corrientes convertido a DEG a fin del periodo.

Fuente: Elaboración propia con base en www.imf.org

\section{$\underline{\text { DeSRaArrollo }}$}


Cuadro A.2

FMI: Detalle de acuerdos aprobados, sep. 08-ago. 09. En millones de DE G. Sólo CRG

\begin{tabular}{|c|c|c|c|c|c|c|c|c|c|c|c|c|c|c|c|}
\hline A & $B$ & $C$ & $D$ & $E$ & $F$ & G & $H$ & 1 & J & K & $L$ & M & N & 0 & $P$ \\
\hline Tipo & Prec & Acceso & País & Fecha & $\begin{array}{c}\text { Duración } \\
\text { meses }\end{array}$ & PIB & Cuota & \begin{tabular}{|c} 
Monto \\
aprobado
\end{tabular} & $\begin{array}{c}\% \mathrm{del} \\
P I B\end{array}$ & $\begin{array}{l}\% \text { de } \\
\text { cuota }\end{array}$ & Desembolsos & \begin{tabular}{|c|} 
Relación \\
L/I
\end{tabular} & $\begin{array}{l}\text { Monto } \\
\text { aprobado } \\
\text { al inicio }\end{array}$ & $\begin{array}{c}\text { Primer } \\
\text { desembolso }\end{array}$ & $\begin{array}{l}\text { Relación } \\
0 / N\end{array}$ \\
\hline SBA & & $E$ & Georgia & $\operatorname{sep} 08$ & 18 & 6472 & 150 & 477 & $7.37 \%$ & $318 \%$ & 288 & $60 \%$ & 477 & 162 & $34 \%$ \\
\hline SBA & & $N E$ & Ucrania & nov 08 & 24 & 89634 & 1372 & 11000 & $12.27 \%$ & $80 \%$ & 7000 & $64 \%$ & 11000 & 3000 & $27 \%$ \\
\hline SBA & & $N E$ & Hungría & nov 08 & 17 & 87553 & 1038 & 10537 & $12.03 \%$ & $1015 \%$ & 7587 & $72 \%$ & 10537 & 4200 & $40 \%$ \\
\hline SBA & & $N E$ & Seychelles & nov 08 & 24 & 451 & 9 & 18 & $4.00 \%$ & $200 \%$ & 8 & $44 \%$ & 18 & 6 & $33 \%$ \\
\hline SBA & & $N E$ & Islandia & nov 08 & 24 & 12800 & 118 & 7400 & $10.94 \%$ & $1186 \%$ & 560 & $40 \%$ & 1400 & 560 & $40 \%$ \\
\hline SBA & & $E$ & Pakistán & nov 08 & 23 & 90977 & 1034 & 7235 & $7.95 \%$ & $500 \%$ & 3403 & $47 \%$ & 5168 & 2067 & $40 \%$ \\
\hline SBA & & $E$ & Letonia & $\operatorname{dic} 08$ & 27 & 17190 & 127 & 1522 & $8.85 \%$ & $1198 \%$ & 714 & $47 \%$ & 1522 & 535 & $35 \%$ \\
\hline SBA & & $N E$ & Belarús & ene 09 & 15 & 28333 & 386 & 2269 & $8.01 \%$ & $588 \%$ & 956 & $42 \%$ & 1620 & 517 & $32 \%$ \\
\hline SBA & Prec & $N E$ & El Salvador & ene09 & 15 & 12892 & 171 & 514 & $3.99 \%$ & $301 \%$ & 0 & $0 \%$ & 514 & 0 & $0 \%$ \\
\hline SBA & & $N E$ & Serbia & ene09 & 27 & 25220 & 468 & 2619 & $10.38 \%$ & $560 \%$ & 701 & $27 \%$ & 351 & 234 & $67 \%$ \\
\hline SBA & & $N E$ & Armenia & mar 09 & 28 & 5840 & 92 & 534 & $9.14 \%$ & $580 \%$ & 264 & $49 \%$ & 368 & 151 & $41 \%$ \\
\hline SBA & & $N E$ & Mongolia & abr 09 & 17 & 2464 & 51 & 153 & $6.21 \%$ & $300 \%$ & 77 & $50 \%$ & 153 & 51 & $33 \%$ \\
\hline SBA & Prec & $N E$ & Costa Rica & $a b r 09$ & 15 & 16600 & 164 & 492 & $2.96 \%$ & $300 \%$ & 0 & $0 \%$ & 492 & 0 & $0 \%$ \\
\hline LCF & Prec & $n / a$ & México & $a b r 09$ & 12 & 647248 & 3153 & 31528 & $4.87 \%$ & $1000 \%$ & 0 & $0 \%$ & 31528 & 0 & $0 \%$ \\
\hline SBA & Prec & $N$ & Guatemala & abr 09 & 18 & 21322 & 210 & 631 & $2.96 \%$ & $300 \%$ & 0 & $0 \%$ & 631 & 0 & $0 \%$ \\
\hline SBA & & $E$ & Rumania & may 09 & 24 & 105036 & 1030 & 11443 & $10.89 \%$ & $1717 \%$ & 4370 & $38 \%$ & 11443 & 4370 & $38 \%$ \\
\hline LCF & Prec & $n / a$ & Polonia & may 09 & 12 & 267103 & 1369 & 13690 & $5.13 \%$ & $1000 \%$ & 0 & $0 \%$ & 13690 & 0 & $0 \%$ \\
\hline LCF & Prec & $n / a$ & Colombia & may 09 & 12 & 128226 & 774 & 6966 & $5.43 \%$ & $900 \%$ & 0 & $0 \%$ & 6966 & 0 & $0 \%$ \\
\hline SBA & & $N$ & Bosnia-Herzeg. & jul 09 & 36 & 9597 & 169 & 1015 & $10.58 \%$ & $600 \%$ & 183 & $18 \%$ & 1015 & 183 & $18 \%$ \\
\hline SBA & & N & Sri Lanka & jul 09 & 20 & 20470 & 413 & 1654 & $8.08 \%$ & $400 \%$ & 207 & $13 \%$ & 1654 & 207 & $13 \%$ \\
\hline
\end{tabular}

Prec: Acuerdo precautorio

Acceso: Excepcional (E) o normal (N)

PIB: valores a finales de 2007. PIB a dólares corrientes convertido a DEG a fin del periodo.

Fuente: Elaboración propia con base en www.imf.org 
Cuadro A.3

Detalle de Staff Reports consultados según país, etapa del acuerdo y fecha de aprobación por parte del Directorio Ejecutivo

\begin{tabular}{|c|c|c|c|c|c|}
\hline País & Suscripción SBA & $7^{a}$ Revisión & $2^{0}$ Revisión & $3^{0}$ Revisión & Total Staff Reports \\
\hline Georgia & $\operatorname{sep} 08$ & $\operatorname{dic} 08$ & mar 09 & ago 09 & 4 \\
\hline Ucrania & nov 08 & may 09 & & & 2 \\
\hline Hungría & nov 08 & mar 09 & & & 2 \\
\hline Seychelles & nov 08 & mar 09 & jun 09 & & 3 \\
\hline Islandia & nov 08 & & & & 1 \\
\hline Pakistán & nov 08 & mar 09 & ago 09 & & 3 \\
\hline Letonia & $\operatorname{dic} 08$ & & & & 1 \\
\hline Belarús & ene 09 & jun 09 & & & 2 \\
\hline El Salvador & ene 09 & & & & 1 \\
\hline Serbia & ene 09 & may 09 & & & 2 \\
\hline Armenia & mar 09 & jun 09 & & & 2 \\
\hline Mongolia & abr 09 & jun 09 & & & 2 \\
\hline Costa Rica & abr 09 & & & & 1 \\
\hline Guatemala & abr 09 & & & & 1 \\
\hline Rumania & $\operatorname{may} 09$ & & & & 1 \\
\hline Bosnia y Herzegovina & jul 09 & & & & 1 \\
\hline Total & & & & & 29 \\
\hline
\end{tabular}

Total acuerdos relevados: 16 SBA (no hay datos sobre el SBA de Sri Lanka).

Total Staff Reports analizados: 29 (16 prerreforma y 13 posrreforma).

Fuente: Elaboración propia con base en IFM staff reports (disponibles en www.imf.org).

Cuadro A.4

FMI: condicionalidades estructurales por área y grado de exigencia, sep. 08-ago. 09 Todas las condicionalidades. Sólo CRG

\begin{tabular}{|l|c|c|c|c|}
\hline & Acción previa & Criterio de ejecución & $\begin{array}{c}\text { Parámetro } \\
\text { de referencia }\end{array}$ & Total \\
\hline Sector financiero & 12 & 10 & 43 & 65 \\
\hline Política cambiaria y monetaria & 14 & 1 & 24 & 39 \\
\hline Gasto público & 13 & 3 & 20 & 36 \\
\hline Política fiscal & 2 & 2 & 13 & 17 \\
\hline Reforma empresas públicas & 1 & 0 & 1 & 2 \\
\hline Privatizaciones & 0 & 0 & 1 & 1 \\
\hline Reforma empleo público & 1 & 0 & 3 & 4 \\
\hline Política social & 1 & 0 & 1 & 2 \\
\hline Otras áreas Banco Mundial & 0 & 0 & 41 & 4 \\
\hline Total & 44 & 16 & 10 & 170 \\
\hline
\end{tabular}

Fuente: Elaboración propia con base en FMI.

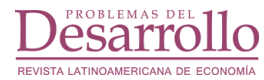

